\title{
Foreword
}

\section{His Bundle Pacing: A 100-Year Journey}

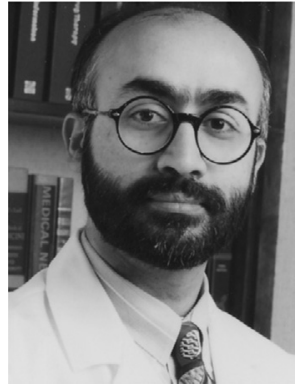

Ranjan K. Thakur, MD, MPH, MBA, FHRS

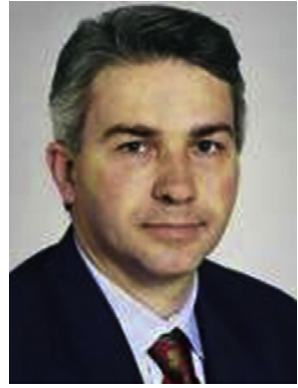

Andrea Natale, MD, FACC, FHRS

\section{Consulting Editors}

We welcome readers to the current issue of Cardiac Electrophysiology Clinics on His bundle pacing. Like many other fields in medicine, the story of His bundle pacing is quite interesting, demonstrating the value of physiological discoveries long forgotten and grit and determination of a lone ranger to champion an idea.

Wiggers $^{1}$ had shown the deleterious hemodynamic effects of ventricular pacing almost 100 years ago. However, when cardiac pacing developed in the 1960s, endocardial ventricular pacing was an obvious way forward. Unfortunately, it took us more than four decades to realize that right ventricular pacing may be harmful, and biventricular pacing was adopted as a method to mitigate the harm.

Dr Deshmukh's contribution to the field of His bundle pacing is to be lauded. Before pacinginduced cardiomyopathy became well appreciated in electrophysiology circles, spurred by the work of Wiggers, the teachings of his own physiology professor in medical school, and the animal work by Dr Scherlag, Dr Deshmukh embarked on clinical His bundle pacing to achieve physiologic ventricular activation more than twenty years ago. The magnitude of this challenge was enormous because there were no special tools available. He used commercially available active-fixation leads and manually formed stylets to direct the tip of the pacing lead to the Hisbundle. An average implant lasted several hours; yet he persisted and his seminal work was published almost two decades ago. ${ }^{2}$ While demonstrating the feasibility of $\mathrm{His}$ bundle pacing resulting in synchronous ventricular activation and hemodynamic benefits, Dr Deshmukh also added to the established criteria for demonstrating selective His bundle pacing. He singularly transformed an idea into clinical reality.

Since his initial publication in 2000 , although adopted slowly, the benefit of His bundle pacing has sparked the imagination of electrophysiologists, and some investigators have made valuable contributions and demonstrated its utility in various circumstances. However, until recently, His bundle pacing was the purview of a few interested electrophysiologists. Recently, there has been an upsurge in interest and widespread diffusion of this technique, evidenced by an increasing number of laboratories starting to use it.

This issue of Cardiac Electrophysiology Clinics focuses on the current utility of His bundle pacing, its uses under various conditions, advantages, and challenges. Going forward, we see His bundle pacing as a preferred site for pacing, whenever it 
can be achieved. But, a lot more needs to be learned yet, and we need better leads and delivery mechanisms to help accomplish it quickly and more reliably. To reach that goal, we will need help and innovation from our colleagues in the pacing industry.

We want to thank Dr Deshmukh and Dr Ellenbogen for editing an excellent issue of Cardiac Electrophysiology Clinics. Many of the contributors are established investigators in the field, and we thank them for their excellent articles. We hope that the readers will find this issue informative and useful in their own journey to delivering physiologic pacing via the His bundle.

Ranjan K. Thakur, MD, MPH, MBA, FHRS Sparrow Thoracic and Cardiovascular Institute Michigan State University 1440 East Michigan Avenue, Suite 400 Lansing, MI 48912, USA
Andrea Natale, MD, FACC, FHRS Texas Cardiac Arrhythmia Institute

Center for Atrial Fibrillation at

St. David's Medical Center 1015 East 32nd Street, Suite 516

Austin, TX 78705, USA

E-mail addresses:

thakur@msu.edu (R.K. Thakur) andrea.natale@stdavids.com (A. Natale)

\section{REFERENCES}

1. Wiggers CJ. The muscular reactions of mammalian ventricles to artificial surface stimuli. Am J Physiol 1925;73:346-78.

2. Deshmukh P, Casavant DA, Romanyshan M, et al. Permanent, direct His-bundle pacing. Circulation 2000;101:869-77. 